\title{
AUTOMATIC RETINAL VESSEL TORTUOSITY MEASUREMENT
}

\author{
${ }^{1}$ Nidhal Khdhair El Abbadi and ${ }^{2}$ Enas Hamood Al Saadi \\ ${ }^{1}$ Department of Computer Science, Education College, University of Kufa, Najaf, Iraq \\ ${ }^{2}$ Department of Computer Science, Education College, University of Babylon, Babylon, Iraq
}

Received 2013-08-11, Revised 2013-08-14; Accepted 2013-09-21

\begin{abstract}
Retinal vascular vessels have the role to indicate the retinal diseases and for systematic diseases when there are any abnormalities in retinal vascular pattern. A characteristic of the vascular pattern that is appreciated by clinicians is vascular tortuosity, i.e., how curved or kinked a blood vessel, either vein or artery, appears along its course. In this study we suggest a novel mask filter to track the blood vessel along its course and measuring the blood vessels tortuosity over the entire human retinal vessel network in fundus eye image, by using the arc to chord ratio. The suggested algorithm tested with straight and curve hand drawing lines and gives high accurate results.
\end{abstract}

Keywords: Tortuosity, Retinal, Retinopathy, Image Processing, Blood Vessel

\section{INTRODUCTION}

Over the last few decades with the advances of computer technology, important studies have been made in computer-aided diagnosis of medical images to improve a clinician's confidence in the analysis of retinal medical images. Investigating retinal medical images using computer analysis methods is of both scientific and clinical importance, as understanding the retinal vascular network may be helpful for improving specific treatments of retinal disorders (Talu, 2013).

Tortuosity is a type of geometrical irregularity that can be defined as a measure of curvature and twists or kinks produced in the vessel course (Joshi et al., 2012). Vascular tortuosity is the result of accumulation of curvature along blood vessel length. The hemodynamic flow in specific vessel affected by the tortuosity of that vessel.

Many types of diseases can be predicated by investigation the retinal blood vessel tortusity, such as hypertensive retinopathy, atherosclerosis and Retinopathy of Prematurity (ROP). Early detection of those diseases can be effectively treated. Or else, the patients may become blindness (Li et al., 2010).

Two factors related to tortuosity, proliferation of blood vessels due to age and blood vessels bending due to degenerative changes of the elastic properties of the blood vessel walls.

It is important to create a quantitative measure of tortuosity which help to make a link between tortuosity and vascular pathologies, especially with computer-aided screening. Lotmar, Freiburghaus and Bracher describe the quantitative tortuosity measurement for the fist time and slightly extended by Bracher. This measurement includes the subdivide the tortuous vessel into a group of single arcs manually. Later many measurments have been suggested for image processing, but it remains unclear which one is most appropriate. A common one is the distance metric defined as the ratio of the vessel length to the chord length between the two end points. Other measures suggested are depend on curvature of the blood vessel axis and on the changing of its direction along the vessel.

Generally the retinal blood vessels are straight or gently curved. The blood vessel tend to be tortuos for some diseases. i.e., they become dilated (due to radial stretching of the vessel) and take on a serpentine path (due to longitudinal stretching). The tortuosity may be local; occurring only in a small region of retinal blood vessels, or it may involve the entire retinal vascular tree (Dougherty, 2011).

Corresponding Author: Nidhal Khdhair El Abbadi, Department of Computer Science, Education College, University of Kufa, Najaf, Iraq 


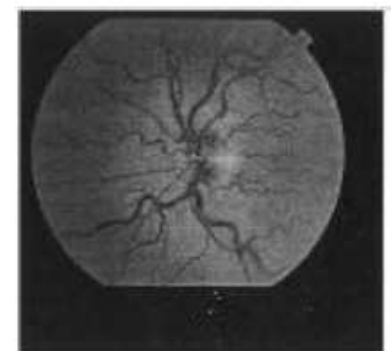

(a)

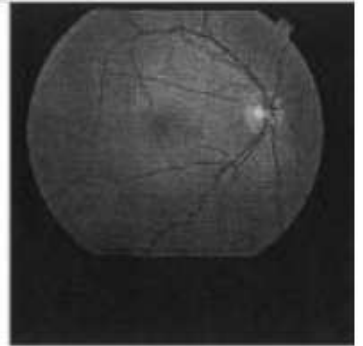

(b)
Fig. 1. (a) Tortuous blood vessel (b) non-tortuous blood vessel

Figure 1 shows images with tortuous and nontortuous blood vessels. Measuring of the blood vessel tortuisty give a good indication about the changing of some diseases with time. Consequently, there is a benefit in measuring tortuosity in a consistent, repeatable fashion. Given the increased availability of digitized fundus photographs, automated tortuosity measurements are now feasible.

High blood flow may occur locally in arteriovenous, anastomosis or systematically in high cardiac output such as accompanies anemia. In response to ischemia or inflammation, new blood vessels grow, often in a destructive manner. Venous congestion can result from the occlusion of the central retinal vein or a branch retinal vein. The resulting elevated intravascular pressure results in tortuosity and dilation in the blocked vein. Vascular tortuosity measurements are commonly performed by human observers using a qualitative scale (e.g., mild, moderate, severe and extreme). Observers may have different grades for the same case due to change the boundaries between observers, also for the same observor will differ with time.

\section{RELATED WORKS}

Onkaew et al. (2011) proposed automatic way to measuring the tortuosity for retinal blood vessels which mainly depend on calculating the curvature from improved chain code algorithm, this process regards the the number of inflection point. The algorithm determine the tortuosity without needed the segmentation of vessel tree. This algorithm classify the blood vessel as tortuous or non-tortuous automaticaly.

Onkaew et al. (2011) introduced a method of how to measure and define tortuous scale using the mean curvature. The tortuosity measurement is calculated base on the radius derive from chord in each curve of the blood vessel. The result obtains from this method can set the standard of tortuosity grading in the retinal vessel. The advantage of this method is to reduce the error in tortuosity calculation, which cause by the low quality image of the retinal vessel.

Dougherty et al. (2010) suggested new relation between tortuisty ranking based on the expert panel of ophthalmologists and the tortusity ranking validity of the mean tortuosity (M) and the normalized root-meansquare tortuosity $(\mathrm{K})$. The tortuosity classifications process highly affected by the low prevalence's of the diseased conditions in the general population and precludes the use of tortuosity for screening for all of these conditions.

In the study of (Kumar and Madheswaran, 2010), suggested to determine the blood vessels thickness automatically. The first step in this algorithm after capturing the imag was to binaries and skeletonised the image to view the blood vesslels network which contain all the structure of blood vessel nodes. The algorithm used the blood vessels segmentation and identification the bifurcations.

Trucco et al. (2010), suggested a method that take in account the vessel thickness as important factor in addition to the curvature which used to determine the tortuosity. This work supported by test it's with 200 different vessels selected by clinical from the public database.

Joshi et al. (2010) introduced a new one dimensional metric (the tourtusity index) which produced from the number of changing in the curvature sign. Vascular tourtusity is determined by dividing the curve length over the cord length.

\section{PROBLEM ISSUES}

Recentally, many researcher works with the computer aided to determine the vessel tortuosity, but few of them working on a fully automated system. Tracing the vessels and finding the length of each branche are two major problems facing the fully automated system.

\section{METHODOLOGY}

We first perform binary skeletonisation for the human retinal vessels network in fundus eye image. After skeletonisation the terminal and branching nodes are then located and removing from the skeleton network; skeletonisation and removing branching nods achieved by using algorithms suggested in the previous papers. This will produce a number of Blood Vessels Segments (BVS), which we work on as shown in Fig. 2. One of the amjor difficulties in tracing is tracing the vessels with high curveture, the difficulties results from the ambiguity of determine the high curvature as vessel branch or vessels tortuosity. Wrong tracers will produce fake tortuosity. 


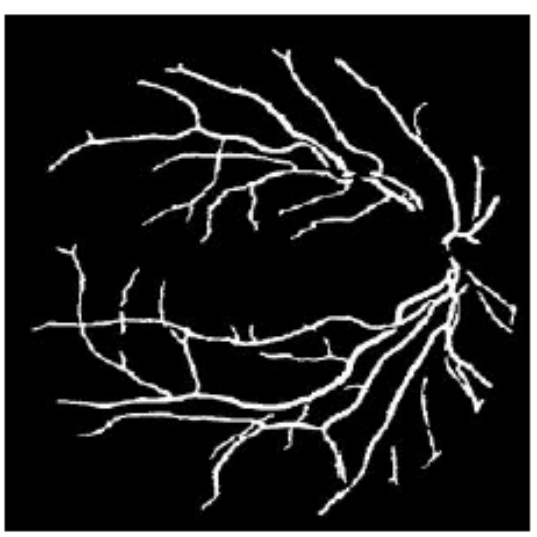

(A)

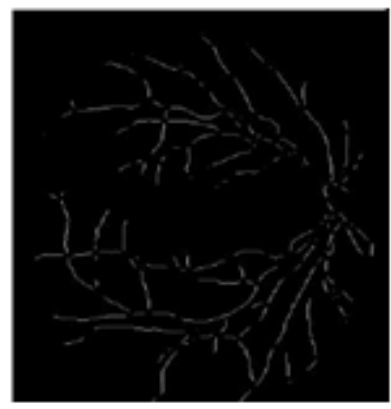

(B)

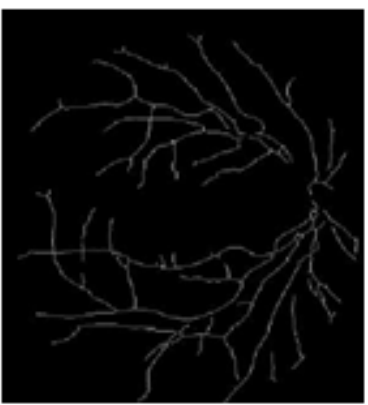

(C)
Fig. 2. (A) Blood vessel network. (B) Blood vessels network skeleton. (C) Blood vessels network skeleton after removing branches and nodes

Every segment in the segmented blood vessels network image is labeled by image label algorithm, this can be accomplished by scan all the pixels in the image, the non zero pixels will be labeled as preliminary labels and recording label equivalences in a union-find table. The union-find algorithm (The Union-Find algorithm is used for maintaining a number of non-overlapping sets from a finite universe of elements) used to resolve the equivalence classes. Finally relabeled the pixels based on the resolved equivalence classes.

Tortuosity is calculated for every labeled blood vessel segment by the following steps:

Step 1: The following mask shown in Fig. 3 is created and suggested to used in this study to check the tortuosity for every blood vessel segment.

Step 2: This mask will be centered on the beginning of one end of each BVS (center element of the mask over the first pixel in the BVS) and pass along the blood vessel until the other end of the segment, its main purpose is to determine the length of this BVS

\begin{tabular}{|r|r|r|}
\hline-7 & -2 & -4 \\
\hline 0 & 0 & 1 \\
\hline 7 & 2 & 4 \\
\hline
\end{tabular}

Fig. 3. Suggested mask
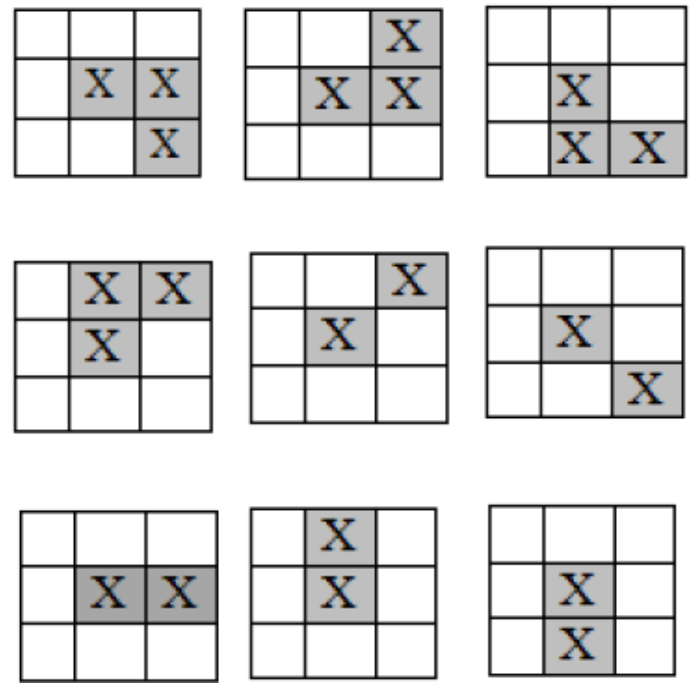

Fig. 4. Probability of blood vessel pixels matched with the mask

When the mask pass along the BVS (always the center of filter move to the next pixel), the orientation of blood vessel segment pixels in regards to mask will be as shown in Fig. 4 (the movement of mask from the left to the right). There are nine possible orientations of BVS pixels according to the filter. Figure 4 shows the BVS pixels in the gray color, while the white color in the mask represent the background pixel in the image (no pixels of BVS match with filter). (Note: when the mask move to the next pixel, the old pixel will be erased).

Step 3: Coordinates of the first pixel and ends one for BVS are determined and stored

Now the mask will be move along the BVS from first point to the end point with one pixel at each step, during each step the algorithm check the orientation of BVS pixels according to the mask and add either 1 or $\sqrt{2}$ to the counter used to determine the BVS length (counter initiate with zero value when the mask centered on the first pixel of BVS) and the current pixel before movement is erased.

This is accomplished by checking the value resulted from matching the mask with BVS, this value determined from the sum of multiplying each point in the 
image by corresponding value in the mask (note: the value of BVS equal one, while the background of image equal zero in skeleton binary image). Let us assign the variable (D) to the value provided from this step.

The value of $\mathrm{D}$ will help us with two subjects:

- First one, its help us to determine the coordinates of the next pixel when the mask move one step, this maintain the movement along the BVS only, (ensure to follow the BVS pixels and keep the next pixel on the center of the filter)

According to the value of (D), the algorithm can estimate the coordinates of the next pixel in the BVS in which the mask will move to it and keeping the mask center on it. The coordinates of the next pixel corresponding to the values of $\mathrm{D}$ are:

- If ( $\mathrm{D}=1\|\mathrm{D}=5\| \mathrm{D}=-3)$ then $\mathrm{y}=\mathrm{y}+1$, $\mathrm{x}$ not change

- If $(D=2 \| D=6)$ then $x=x+1$, y not change

- If ( $D=-2 \| D=-6)$ then $x=x-1$, y not change

- If $(D=0)$ then $y=y-1, x$ not change

- If $(D=-4)$ then $x=x-1$ and $y=y+1$

- If $(D=4)$ then $x=x+1$ and $y=y+1$

- If $(D=7)$ then $x=x+1$ and $y=y-1$

- If $(D=-7)$ then $x=x-1$ and $y=y-1$

Counting the length of BVS can be done depending on the value of $\mathrm{D}$, as following:

If $(\mathrm{D}=4\|\mathrm{D}=-4\| \mathrm{D}=7 \| \mathrm{D}=-7)$ then

count $=$ count $+\sqrt{2}$

Else count $=$ count +1

The variable count ultimately will be equal to the length of BVS when the mask reaches the last pixel in the BVS.

The arc to chord ratio need two factors to find the tortuisty, the first one is the length of BVS which calculated previously with variable (count), while the second one is the straight distance $\left(D_{\text {straight }}\right)$ between the first point and last point in BVS. The straight distance $\left(\mathrm{D}_{\text {straight }}\right)$ is calculated using Euclidean distance as follow:

$$
\mathrm{D}_{\text {straight }}=\sqrt{(\mathrm{x} 2-\mathrm{x} 1)^{2}+(\mathrm{y} 2-\mathrm{y} 1)^{2}}
$$

where, $(\mathrm{x} 1, \mathrm{y} 1)$ are the coordinates of the first point in the BVS and $(\mathrm{x} 2, \mathrm{y} 2)$ are the coordinates of second point in the BVS.

The tortuosity of the BVS is defined as the ratio of the Count to the $\mathrm{D}_{\text {straight }}$ :

$$
\text { Tortuosity }=\frac{\text { Count }}{D_{\text {straight }}}
$$

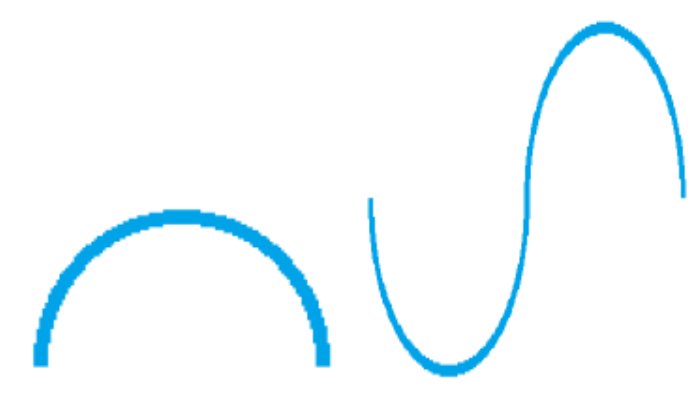

Fig. 5. Examples of lines with predefined lengths, used to test the suggested algorithm

The suggested algorithm tested with many hand drawing (straight lines and curves) as shown in Fig. 5, which they have predefined lengths, the results was very accurate for both lengths determined and tracing the curve from the first end until reach the other end.

\section{CONCLUSION}

This paper present a fully automated tortuosity diagnosis system of retinal images. Our system suggested new mask to resolve two important issues includes: (1) Identify and tracing each branch of retinal blood vessels automatically. (2) Measuring the tortuosity by using arc and chord ratio.

Tested of this system gives promise and highly accurate results.

\section{REFERENCES}

Dougherty, G., 2011. Medical Image Processing. 1st Edn., Techniques and Applications, Springer, New York, ISBN-10: 1441997792, pp: 395.

Dougherty, G., M.J. Johnson and M.D. Wiers, 2010. Measurement of retinal vascular tortuosity and its application to retinal pathologies. Med. Biol. Eng. Comput., 48: 87-95. DOI: 10.1007/s11517-009-0559-4

Joshi, V., R.M. Joseph and M.D. Abramoff, 2010. Automated measurement of retinal blood vessel tortuosity. Proceedings of the SPIE Medical Imaging: Computer-Aided Diagnosis, (CAD' 10), SPIE, pp: 76243A-76251A. DOI: 10.1117/12.844641

Joshi, V.S., 2012. Analysis of retinal vessel networks using quantitative descriptors of vascular morphology. PhD Thesis, University of Iowa. 
Kumar, S.J.J. and M. Madheswaran, 2010. Automated thickness measurement of retinal blood vessels for implementation of clinical decision support systems in diagnostic diabetic retinopathy. World Acad. Sci., Eng. Technol., 40: 393-397.

Li, Q., J. You, J. Wang and A. Wong, 2010. A fully automated system for retinal vessel tortuosity diagnosis using scale dependent vessel tracing and grading. Proceedings of the IEEE 23rd International Symposium on Computer-Based Medical Systems (CBMS), Oct. 12-15, IEEE Xplore Press, Perth, WA 221-225. DOI: 10.1109/CBMS.2010.6042645

Onkaew, D., R. Turior, B. Uyyanonvara, N. Akinori and C. Sinthanayothin, 2011. Automatic retinal vessel tortuosity measurement using curvature of improved chain code. Proceedings of the International Conference on Electrical, Control and Computer Engineering (INECCE), Jun. 21-22, IEEE Xplore Press, Pahang, pp: 183-186. DOI: 10.1109/INECCE.2011.5953872
Talu, S., 2013. Characterization of retinal vessel networks in human retinal imagery using quantitative descriptors. Int. J. Bioflux Soc., 5: 52-57.

Trucco, E., H. Azegrouz and B. Dhillon, 2010. Modeling the tortuosity of retinal vessels: Does caliber play a role? IEEE Trans. Biomed. Eng., 57: 2239-2247. DOI: 10.1109/TBME.2010.2050771 